EOMmun Communication et organisation

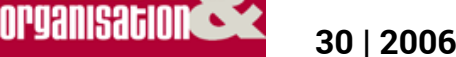

Modèles et modélisations, pour quels usages ?

\title{
Modèles et modélisations, pour quels usages
}

Valérie Carayol et Gino Gramaccia

\section{OpenEdition}

Journals

Édition électronique

URL : http://journals.openedition.org/communicationorganisation/3441

DOI : 10.4000/communicationorganisation.3441

ISSN : $1775-3546$

Éditeur

Presses universitaires de Bordeaux

Édition imprimée

Date de publication : 1 octobre 2006

Pagination : $7-10$

ISSN : 1168-5549

\section{Référence électronique}

Valérie Carayol et Gino Gramaccia, «Modèles et modélisations, pour quels usages », Communication et organisation [En ligne], 30 | 2006, mis en ligne le 21 juin 2012, consulté le 21 septembre 2020. URL http://journals.openedition.org/communicationorganisation/3441 ; DOI : https://doi.org/10.4000/ communicationorganisation.3441

Ce document a été généré automatiquement le 21 septembre 2020

(C) Presses universitaires de Bordeaux 


\title{
Modèles et modélisations, pour quels usages
}

\author{
Valérie Carayol et Gino Gramaccia
}

1 Le présent dossier de Communication \& Organisation traite du statut des modélisations de la communication, des orientations qu'elles empruntent et des usages qui en sont fait, en particulier dans le champ de la communication des organisations. Si les modèles de communication dans les organisations rendent possible la réflexivité, donc l'évolution de l'action collective, alors ils constituent des points d'ouverture scientifiquement multiples pour l'analyse des phénomènes collectifs.

2 Si on peut, bien sûr, discuter du rôle et du statut des pratiques, dès lors que l'on théorise ou modélise, se profile généralement un usage du modèle ou de la théorie produite. Elucidé, revendiqué, mais aussi parfois latent ou associé à un projet de connaissance encyclopédique ou "fondamentale ", le modèle est déjà en soi un objet pour communiquer. Son objet ou son projet peut être scientifique ou heuristique, parfois pédagogique. Il peut avoir des vertus de médiation, de vulgarisation. Des usages dans des domaines aussi variés que l'évaluation, la simulation, la prospective, l'accompagnement de l'action, la prévention peuvent, par exemple, être recensés.

3 L'usage du modèle peut être détourné de son contexte de production initial par des utilisateurs zélés. C'est ainsi que Paul Watzlawick avait, à l'occasion de la remise d'un doctorat Honoris causa de l'Université de Bordeaux 3, raconté comment ses concepts avaient servi à légitimer un certain nombre de pratiques et étaient revendiqués par des praticiens avec lesquels ils n'entretenaient aucune affinité.

4 Si l'analyse des présupposés, des hypothèses et des cheminements qui ont conduit à l'élaboration des modèles est de l'ordre de la réflexion épistémologique, l'analyse de leurs statuts et de leurs usages, notamment dans le champ de la communication des organisations constitue un terrain d'investigation encore peu exploré.

5 Dans ce numéro de Communication \& Organisation, les différents contributeurs du dossier s'interrogent sur la notion de modèle, de modélisation, son statut épistémologique ou son statut pragmatique, dans des univers professionnels différents. 
6 Alex Mucchielli montre la diversité des options que recouvre la notion de modèle en insistant sur ce qui en constitue la fonction principale: guider l'observation scientifique à partir de sa théorie de référence. La construction d'un objet scientifique repose sur une totalité paradigmatique intégrant la posture épistémologique du chercheur, la théorie et la méthode. Rappelant les principes de la modélisation systémique des communications, l'auteur insiste sur les conditions dans lesquelles de tels principes doivent être appliqués et en premier lieu, celle qui impose que soit dégagé le caractère à la fois observable et construit du phénomène communicationnel. Est rappelé ce postulat essentiel selon lequel la connaissance, pour le constructivisme, se ramène au processus de sa propre construction : d'où la nécessité de méthodologies itératives et modulables (souplesse des grilles d'analyse, par exemple), particulièrement utiles à l'étude des situations organisationnelles.

7 Christian Le Moënne souligne l'ambiguïté heuristique de la notion de modèle. Le modèle permet à la fois d'expliquer et d'anticiper l'action - et dans ce cas, nous sommes dans une logique pragmatique de succès de l'agir - tandis que, dans la perspective de la découverte scientifique, il se réduit à une construction symbolique livrant une forme achevée du raisonnement. L'auteur dépasse cette ambiguïté par cette question: comment monter en généralité et fonder une connaissance et des langages communs? Cette étude montre qu'une convergence entre les deux perspectives est possible à la lumière de l'approche pragmatique dans l'hypothèse où «toute connaissance est transformation active des modèles et thèmes existants ». En sorte que la modélisation pragmatique serait alors une heuristique.

8 Pour Gilles Le Cardinal et Jean-François Guyonnet, la modélisation est une posture de recherche délicate qui impose l'étude de fondements épistémologiques clairs, pour qu'elle puisse être conçue, mise en oeuvre et transmise de façon pertinente et utile, notamment en recherche-action. Il s'agit d'expliciter les conditions à réunir, les questions auxquelles il faut répondre et les étapes de la démarche de modélisation d'un système complexe, qu'elle soit réalisée seul ou en équipe. Ils concluent sur les liens récursifs qui unissent le modélisateur, la modélisation et le modèle, en insistant sur le fait que l'acte de modélisation a une influence sur le modélisateur.

Dans le cas de la recherche-action, la modélisation devient un élément directement constitutif - voire réflexif - de l'activité de recherche. L'une des questions soulevées, à ce propos, par Vincent Meyer, porte sur la manière dont les individus peuvent devenir les «savants de leur propre réalité ». L'auteur confirme la place centrale que doivent occuper les Sciences de l'information et de la communication dans une telle démarche, notamment dans l'analyse des enjeux managériaux contemporains. Se pose également la question de la légitimité - de l'autorité - du savoir issu de la recherche-action en regard du discours savant. La réponse est assurément liée à la reconnaissance d'une nécessaire dialectique opposant et rapprochant, dans le même débat, l'auteur et le chercheur.

10 Miguel de Aguilera retrace l'histoire scientifique de la communication en soulignant l'influence des conditions déterminantes dans la genèse de ses objets. L'expansion des systèmes de communication "de masse» explique, dans la société industrielle, l'importance du paradigme informationnel comme principe organisateur de recherches en communication. Mais, comme l'explique l'auteur, le tournant culturel marque l'obsolescence de ce modèle devant l'irruption de la question du sens. La sémiotique, les 
Cultural Studies, deviennent alors des disciplines annoncées pour des champs d'expérimentation nouveaux et des pratiques modélisatrices émergentes.

11 La création publicitaire constitue assurément un champ d'action privilégié pour tester la validité de constructions modélisatrices à l'heure où, comme l'affirme Arlette Bouzon, les recherches scientifiques sur ces pratiques sont rares. L'auteur part d'une hypothèse originale : le créatif, en publicité, est un bricoleur et un "braconneur » de modèles. C'est une critique du bricolage et du braconnage dans la fabrication des messages publicitaires qui est ici proposée, montrant en quoi les théories de référence interviennent en premier lieu comme des types explicatifs de pratiques consuméristes. Si les sciences humaines sont convoquées par les concepteurs, c'est au titre de l'efficacité de "modèles réduits " à la simplicité du type. Le modèle est tout à la fois l'outil de conception des messages, la référence théorique explicative des pratiques de consommateurs et sans doute l'un des facteurs de changement de ces pratiques. C'est sans doute ce qui explique la créative désinvolture des concepteurs, empressés d'emprunter mais toujours efficaces puisqu'ils parviennent toujours à produire le juste nécessaire dans le cadre de stratégies de séduction spécifiques et certainement éphémères.

Ce point de vue, repris Bernard Dagenais, est vérifié dans le domaine des relations publiques. Les modèles qu'utilisent les différentes disciplines de la connaissance humaine sont empreints d'effets de mode. En communication, certains modèles s'imposent, comme celui de Shannon et Wiener, et d'autres ont une courte vie comme celui de Moles (la dynamique socio-culturelle) et Morin (le modèle génétique). Les relations publiques ont créé peu de modèles qui leur soient propres, parmi lesquels le modèle RACE. Mais elles ont aussi été hermétiques à des modèles très efficaces, comme les méthodes du média planning.

13 Par quels circuits et courants d'idées les modèles d'organisation trouvent-ils de fertiles terrains d'applications? Lucia Granget et Hugues Draelants abordent cette question à propos de l'université et d'établissements scolaires. L'idée d'une université « entrepreneuriale » émerge, mais elle est confrontée aux freins traditionnels qu'opposent la bureaucratisation aux processus décisionnels ou encore la dissémination des pôles de décision. Lucia Granget souligne cependant que les conditions à l'introduction d'un modèle global de marketing dans l'institution universitaire sont maintenant réunies et ce, compte tenu des enjeux liés à l'internationalisation et aux techniques nouvelles de la transmission des connaissances. Hugues Draelants discute de la pertinence d'une importation du concept d'identité organisationnelle depuis le domaine de la théorie des organisations et $\mathrm{du}$ management, où ce concept connaît actuellement un certain succès, dans le domaine de la sociologie de l'éducation et des établissements scolaires. Compte tenu du statut théorique peu fondé de l'établissement comme catégorie sociologique, l'auteur envisage la pertinence d'un tel transfert. Il discute les conditions socio-politiques, institutionnelles et organisationnelles nécessaires au développement d'identités organisationnelles dans le cas des établissements scolaires, en montrant notamment que l'évolution actuelle des politiques et modes de régulation de l'enseignement favorisent la différenciation entre écoles et donc la production d'identités d'établissements. 